\title{
Introduction
}

\section{Low-grade gliomas}

Mitchel S. Berger, M.D., ${ }^{1}$ And Bob S. Carter, M.D., Ph.D. ${ }^{2}$

\footnotetext{
${ }^{1}$ Department of Neurosurgery, University of California, San Francisco; and ${ }^{2}$ Department of Neurosurgery, University of California, San Diego, La Jolla, California
}

This issue of Neurosurgical Focus is particularly notable for its emphasis in 2 areas: the burgeoning interest in the genomics of glioma and the ongoing interest in improving the technical aspects of surgery for this disease.

This issue leads with excellent reviews by Marko and Weil and Dunn et al., who respectively provide an overview of the genetic changes observed in low-grade gliomas (LGGs) as well as an in-depth review of IDH1 mutation pathophysiology. As a prototypical tumor-specific genetic change, the IDH1 mutation has important implications for prognosis, therapeutic decision making, and use as a biomarker in LGG. An additional article by Bagley et al. features a study of information stored in a large database (Surveillance Epidemiology and End Results [SEER]) that provides insights on LGG survival.

Rey-Dios and Cohen-Gadol, Wilden et al., Talacchi et al., and Rapp et al. focus on the technical aspects of LGG surgery and include a video that should be of particular interest to any who are focused on the technical nuances of achieving maximal tumor resection.

Finally, the issue concludes with a highlighted overview from the 11th Annual Frye-Halloran Symposium held at the Massachusetts General Hospital in the autumn of 2012, which brought together a number of leading experts in the molecular genetics of LGG, pointing out the emerging excitement of using genetic information to guide therapeutic decision making.

We anticipate that you will find this issue to be useful in the practice of neurosurgical oncology today as well as informative regarding key directions for research in LGG going forward.

(http://thejns.org/doi/abs/10.3171/2012.12.FOCUS12407)

\section{Disclosure}

The authors report no conflict of interest.
Please include this information when citing this paper: DOI: 10.3171/2012.12.FOCUS12407. 